\title{
Optogenetic Excitation of Central Amygdala Amplifies and Narrows Incentive Motivation to Pursue One Reward Above Another
}

\author{
${ }^{\circledR}$ Mike J.F. Robinson, ${ }^{1,2}{ }^{\circledR}$ Shelley M. Warlow, ${ }^{1}$ and ${ }^{\circledR K e n t ~ C . ~ B e r r i d g e ~}{ }^{1}$ \\ ${ }^{1}$ Psychology Department, University of Michigan, Ann Arbor, Michigan 48109, and 2Psychology Department, Wesleyan University, Middletown, \\ Connecticut 06459
}

\begin{abstract}
Choosing one reward above another is important for achieving adaptive life goals. Yet hijacked into excessive intensity in disorders such as addiction, single-minded pursuit becomes maladaptive. Here, we report that optogenetic channelrhodopsin stimulation of neurons in central nucleus of amygdala (CeA), paired with earning a particular sucrose reward in rats, amplified and narrowed incentive motivation to that single reward target. Therefore, CeA rats chose and intensely pursued only the laser-paired sucrose reward while ignoring an equally good sucrose alternative. In contrast, reward-paired stimulation of basolateral amygdala did not hijack choice. In a separate measure of incentive motivation, CeA stimulation also increased the progressive ratio breakpoint or level of effort exerted to obtain sucrose reward. However, CeA stimulation by itself failed to support behavioral self-stimulation in the absence of any paired external food reward, suggesting that CeA photo-excitation specifically transformed the value of its external reward (rather than adding an internal reinforcement state). Nor did CeA stimulation by itself induce any aversive state that motivated escape. Finally, CeA stimulation also failed to enhance 'liking' reactions elicited by sucrose taste and did not simply increase the general motivation to eat. This pattern suggests that CeA photo-excitation specifically enhances and narrows incentive motivation to pursue an associated external reward at the expense of another comparable reward.
\end{abstract}

Key words: central amygdala; motivation; optogenetics; reward

\section{Introduction}

Amygdala-related circuitry plays an important role in motivation and learning, including incentive motivation for rewards as well as fear of threats (Baxter and Murray, 2002; Will et al., 2004; Balleine and Killcross, 2006; LeDoux, 2007; Mahler and Berridge, 2009; DiFeliceantonio and Berridge, 2012; Pelloux et al., 2013). Learning a preference for one particular reward is often adaptive in guiding normal choice and pursuit. However, pathological amplification and narrowing in the focus of incentive motivation may produce drug addiction, binge eating, gambling, or related compulsive pursuit disorders. When single-minded pursuit of an addictive reward occurs at the expense of other life goals, such a winner-take-all narrowing of motivation preference may involve dysfunction in brain circuitry involving the amygdala (Schneider et al., 2001; Makris et al., 2004; Wrase et al., 2008; Lesscher and Vanderschuren, 2012).

Received May 18, 2014; revised Sept. 28, 2014; accepted 0ct. 6, 2014.

Author contributions: M.J.F.R. and K.C.B. designed research; M.J.F.R. and S.M.W. performed research; M.J.F.R. and S.M.W. analyzed data; M.J.F.R., S.M.W., and K.C.B. wrote the paper.

This work was supported by the National Institutes of Health (Grants DA015188 and MH63649 to K.C.B.). We thank Aaron Garcia, Adam Fischer, Daniel Miller, and Cody Schember for technical assistance and histology and Marc Bradshaw and Christopher Broussard for constructing equipment.

The authors declare no competing financial interests.

Correspondence should be addressed to Mike Robinson, Psychology Department, Wesleyan University, 207 High Street, Middletown, CT 06459. E-mail: mjrobinson@wesleyan.edu.

DOI:10.1523/JNEUROSCI.2013-14.2014

Copyright $\odot 2014$ the authors $\quad 0270-6474 / 14 / 3416567-14 \$ 15.00 / 0$
Within the amygdala, the basolateral nucleus (BLA) and central nucleus (CeA) are arranged partly in series, but also each possess independent inputs and outputs that allow the two nuclei to act in parallel (Killcross et al., 1997; Parkinson et al., 2000; Will et al., 2004; Corbit and Balleine, 2005; Balleine and Killcross, 2006; LeDoux, 2007; Mahler and Berridge, 2009; DiFeliceantonio and Berridge, 2012; Lingawi and Balleine, 2012; Roesch et al., 2012). Here, we used optogenetic stimulation in CeA versus BLA to compare their roles (Tye et al., 2011) and reveal new aspects of amygdala-related control of incentive motivation and focused pursuit. Our results reveal a CeA mechanism able to both narrow and amplify learned incentive motivation in an addictive-like fashion. 'Wanting' to pursue one reward that is associatively paired with CeA stimulation becomes specifically amplified, even at the expense of earning another alternative reward of comparable value.

\section{Materials and Methods}

Animals

Female Sprague Dawley rats (250-325 g, $n=31$ ) were housed in a reverse $12 \mathrm{~h} \mathrm{light/dark} \mathrm{cycle} \mathrm{at} 21^{\circ} \mathrm{C}$ constant temperature. Purina chow pellets (15-20 g per day) and ad libitum water were provided. The University Committee on the Use and Care of Animals of the University of Michigan approved all experimental methods performed in this research.

\section{Surgery}

Rats were anesthetized with ketamine (100 mg/kg, i.p.) and xylazine (7 $\mathrm{mg} / \mathrm{kg}$, i.p.), and given atropine $(0.04 \mathrm{mg} / \mathrm{kg}$, i.p. $)$ to protect respiration. 
Each rat was surgically infused bilaterally into the central nucleus of amygdala (CeA rats; $n=$ 14) or the basolateral nucleus of amygdala (BLA rats; $n=8$ ) with $2 \mu$ l of a CAG channelrhodopsin virus (AAV5-CAG-ChR2-GFP) or with an optically inactive control virus lacking ChR2 as inactive-virus control rats (AAV5CAG-GFP; $n=5$ ). Finally, an additional set of control rats received no virus microinjection to serve as a baseline control group $(n=4)$. Bilateral infusions were made through microinjection cannula stereotaxically aimed at $C e A$ : A/P: $-2.4 ; \mathrm{M} / \mathrm{L}: 4 ; \mathrm{D} / \mathrm{V}:-7.6$ (with mouth bar set at -3.3 ) or at BLA: A/P: $-1.6 ; \mathrm{M} / \mathrm{L}: 4.9 ; \mathrm{D} / \mathrm{V}$ : -7.8 , infused over $10 \mathrm{~min}$ at constant rate $(0.2$ $\mu \mathrm{l} / \mathrm{min}$ ), followed by an additional $10 \mathrm{~min}$ in place for diffusion. Bilateral optic fibers (200 $\mu \mathrm{m})$ were also implanted in either central amygdala or basolateral amygdala during the same surgery, aimed $0.2 \mathrm{~mm}$ dorsal to the location of virus injection. Rats were postoperatively treated with the antibiotic chloramphenicol ( $60 \mathrm{mg} / \mathrm{kg}$, s.c.) and carprofen (5 mg/ $\mathrm{kg}$, s.c.) as an analgesic and given at least 3 weeks to recover and to allow for optimal virus expression.

\section{Apparatus}

Instrumental training in the two-sucrose choice test and in the progressive ratio onechoice test was performed in Med-Associates chambers $(30.5 \times 24.1 \times 21.0 \mathrm{~cm})$ with clear Plexiglas floors (Fig. 1). The chamber walls were equipped with 4 illuminated retractable levers $(4.5 \times 2 \mathrm{~cm})$, auditory speakers (for tone/white noise components of CSs), and a magazine for sucrose pellet delivery. Two contact liquid sippers (located on the back wall) and a grid floor were inserted into the chambers for self-stimulation tests and removed when not in use. A video camera placed below the transparent chamber floor recorded the animal's behavior.

Optogenetic self-stimulation tests (without sucrose or other food rewards) were conducted in separate chambers. The first self-stimulation test used a novel place-preference versus place-avoidance measure using a Sensorat apparatus designed in-house, which allowed rats to selfadminister CeA laser illumination by going to a particular place (constituting $1 / 4$ of the chamber area) or to avoid illumination by remaining in any other place within the chamber (i.e., $3 / 4$ of the chamber). The apparatus consisted of a 4-corner square Plexiglas chamber with bedding on the floor $(38 \times 38 \mathrm{~cm})$ and a Plexiglas cylinder that occluded the center (20 cm diameter) so that rats could be only in the periphery or corners. Each of the four corners contained its own motion detector $46 \mathrm{~cm}$ above the floor to sense if the rat entered its location below, with all data recorded by a Matlab computer program. Laser illumination was always paired with entry into one corner (either a $3 \mathrm{~s}$ pulse or $8 \mathrm{~s}$ pulse for different rats; $25 \mathrm{~Hz} ; 8-10 \mathrm{~mW}$ ) serially reactivated by any continuing movement within that corner and terminated by exit from that corner. The same corner was always used for a given individual rat, but different rats were each assigned to their own arbitrarily chosen corners.

The second self-stimulation test allowed rats to self-administer CeA laser stimulation by simply touching a particular object (an empty water spout). The apparatus used 2 metal spouts that protruded from the wall $\sim 5 \mathrm{~cm}$ apart, both empty but physically identical to drinking spouts familiar to rats from their home cage. Touching of 1 designated spout earned brief laser illumination of the rat's amygdala (either a $1 \mathrm{~s}$ or $8 \mathrm{~s}$ pulse for different rats; $25 \mathrm{~Hz} ; 8-10 \mathrm{~mW}$ ) on an FR1 schedule. Touching of the other spout earned nothing. Assignment of spouts as laser paired or inactive control was balanced across different rats.

Taste reactivity testing was performed in a square Plexiglas chamber $(26 \times 27 \times 41 \mathrm{~cm})$ with a transparent Plexiglas floor mounted above a tilted mirror to allow video recording of the animal's orofacial reactions.
Sucrose pellets were delivered slowly one at a time while positive hedonic orofacial reactions were recorded during voluntary consumption.

\section{Procedure}

Making an equal choice unequal: instrumental choice of laser + sucrose versus sucrose alone. During instrumental training, rats (CeA: $n=14$; BLA: $n=8$; control virus: $n=5$ ) were presented two illuminated levers, one on either side of the magazine (Fig. 1). Depressions of one lever (Laser + Sucrose lever) led to instrumental delivery of a sucrose pellet plus $8 \mathrm{~s}$ of $25 \mathrm{~Hz}$ ( $15 \mathrm{~ms} \mathrm{ON}, 25 \mathrm{~ms}$ OFF) blue ( $473 \mathrm{~nm}$ ) laser stimulation at $8-10 \mathrm{~mW}$, accompanied by a distinctive $8 \mathrm{~s}$ auditory cue (white noise or tone; always the same paired with this outcome for a particular rat, but counterbalanced assignments across rats). In contrast, pressing the other lever (Sucrose Alone lever) delivered a single sucrose pellet accompanied by its own distinct $8 \mathrm{~s}$ auditory cue (tone or white noise; whichever was not paired with laser-sucrose), but no laser illumination. For both levers, presses during the $8 \mathrm{~s}$ after sucrose delivery had no further consequence. After $2 \mathrm{~d}$ of initial acquisition, each daily session began with a single lever presented alone to allow opportunity to earn its associated reward (either Laser + Sucrose or Sucrose Alone), after which the lever was retracted. Then, the alternative lever was presented by itself to allow opportunity to earn the other reward. Each lever was presented again alone for a second cycle to ensure that the rat sampled both reward outcomes. Those singlechoice exposures were intended to help the rat learn the association between each lever and its particular outcome. Finally, both levers together were extended for the remainder of the session (30 min total), allowing the rat to freely choose between the two levers and to earn respective rewards in any ratio it chose. In addition, a third lever (inactive control) was constantly extended from the opposite back wall, on which presses earned nothing and simply served as a control measure of general activity that resulted in lever responses. Whenever the number of lever presses required by a day's schedule was completed on either lever (FR1, FR4, RR4, RR6), its sucrose pellet was immediately delivered, accompanied by $8 \mathrm{~s}$ of the appropriate auditory cue that labeled the particular lever and its outcome (white noise or tone). For the Laser + Sucrose lever, delivery of the sucrose pellet was also accompanied by additional simultaneous laser stimulation ( $8 \mathrm{~s}$ pulse; $25 \mathrm{~Hz} ; 8-10 \mathrm{~mW}$ ). During those $8 \mathrm{~s}$, 
animals typically rapidly retrieved the sucrose pellet and then resumed responding on either of the two active levers.

Two separate groups of rats were trained either in this way: one on an increasing schedule of responding effort (FR1 $\rightarrow$ RR6; CeA: $n=10$; BLA: $n=8$ ) and the second simply maintained on a minimal FR1 effort requirements $(n=4)$ for the same number of days to assess the contribution of effort versus accumulating days of experience in the development of a laser preference.

Progressive ratio: Laser + Sucrose versus Sucrose Alone. On day 9, a progressive ratio test was given with either the Laser + Sucrose instrumental lever together with CeA illumination or with the Sucrose Alone instrumental lever without any laser (order of test conditions was balanced across rats). On day 10, the progressive ratio test was repeated for each rat with its other lever and other laser condition (CeA: $n=7$; control virus: $n=5$ ). The number of presses required to produce the next reward delivery increased after each reward, according to an exponential progression (progressive ratio schedule $=1,2,4,6,9,12,15,20,25,32,40$, $50,62,77,95,118,145,178,219,268, \ldots)$ derived from the formula PR $=$ $\left[5 e^{(\text {reward number } \times 0.2)}\right]-5$ and rounded to the nearest integer (Richardson and Roberts, 1996; Saunders and Robinson, 2011). To determine whether any preference in responding was the result of increased workload, animals were given a final FR1 session on day 11, identical to the initial day of training.

Making an unequal choice equal: Laser + Salt versus Sucrose or Salt Alone. To further examine the control of instrumental choice, we investigated whether CeA laser stimulation could make a normally less preferred outcome (salty food pellet adulterated with $3 \% \mathrm{NaCl}$ ) become equally chosen as a normally more preferred outcome (sucrose pellet). Rats were given a choice between two instrumental levers on contiguous days between days 24 and 36 . One lever earned a sucrose pellet alone (Sucrose Alone), as above (RR6). The other lever earned simultaneous CeA laser stimulation (Laser + Salt) plus a normally less-preferred salty pellet $(3 \% \mathrm{NaCl})$. These $30 \mathrm{~min}$ sessions were repeated daily for 6 consecutive days. On the seventh day, the remaining sucrose reward was also replaced by another $3 \% \mathrm{NaCl}$ pellet (but alone, without laser; Salt Alone), so that rats were asked to choose between earning two equally salty pellets, one with CeA stimulation and one without (Laser + Salt vs Salt Alone). This was repeated for $5 \mathrm{~d}$ and followed by $2 \mathrm{~d}$ of the initial Sucrose Alone versus Laser + Sucrose choice to confirm reliability of pattern. Finally, to assess the role of laser stimulation on the less preferred salty pellet outcome, rats were given $6 \mathrm{~d}$ of the same initial instrumental choice (salty pellet vs sucrose pellet), but this time in the absence of any CeA laser stimulation (Salt Alone vs Sucrose Alone). This was followed by at least two days of the initial Sucrose Alone versus Laser + Sucrose instrumental choice, with laser returned to its associated lever/outcome.

Persistence of choice preference in the absence of CeA laser stimulation. In a test of the persistence of CeA ChR2 laser-induced pursuit, we investigated whether it would decay or persist if laser were suddenly turned off after it was well established. Rats that had received $2 \mathrm{~d}$ refresher training with Laser + Sucrose versus Sucrose Alone above were therefore given another 4 consecutive days of laser-extinction testing between days 44 and 47, where outcomes for both levers consisted of a sucrose pellet with its lever-appropriate auditory cue, but no laser stimulation was administered even for the lever-sound option that had earlier been paired with CeA illumination (Sucrose NO Laser vs Sucrose Alone).

Lever self-stimulation in the absence of sucrose: Laser Alone versus No Reward. To conversely assess whether laser stimulation alone could maintain responding on a sucrose-laser-associated lever when actual sucrose reward was discontinued, rats were given the opportunity to earn the same levers but without sucrose (Sucrose Extinction) between days 12 and 18. In a sense, this was a third form of laser self-stimulation test, but using an already established instrumental response that had been learned to earn Laser + Sucrose combinations. It simply investigated whether central amygdala laser alone was sufficient to maintain that acquired response when sucrose was discontinued. Each completed trial (RR4) on the laser lever resulted in delivery of laser stimulation and the previously paired auditory cue but no sucrose pellet delivery (Laser-No Sucrose). Each completed trial on the other lever (previously Sucrose Alone) resulted in delivery of its auditory cue (again without sucrose: No
Sucrose, No Laser). Lever test sessions lasted for $30 \mathrm{~min}$ and were repeated for 7 consecutive days. A separate group of rats was run on Laser + Sucrose extinction, where responses on either lever resulted in delivery of its auditory cue alone (No Laser-No Sucrose versus No Sucrose) to determine whether laser stimulation was responsible for maintaining a preference or any amount of responding.

Laser self-stimulation (CeA laser self-administration without sucrose). Rats that had shown CeA ChR2 incentive facilitation (plus control CeA inactive-virus rats) were allowed to self-administer laser illumination by performing a simple new response in two situations. In the first, by going to a particular corner location in a 4-corner chamber, the rat could earn pulses of CeA laser illumination: an easy way to gain photo-excitation that requires no additional active behavioral responses, which was modeled on the original Olds and Milner demonstration of electrode selfstimulation by going to a location (Olds and Milner, 1954). Rats were placed in a Plexiglas arena $(38 \mathrm{~cm} \times 38 \mathrm{~cm}$; height: $47 \mathrm{~cm})$ in which they could wander the periphery and enter any of 4 corners. The center of the arena was occluded by a Plexiglas cylinder $(20 \mathrm{~cm}$ diameter $\times 30 \mathrm{~cm}$ height) to restrict rats to the outer rim. Each corner of the chamber had its own motion detector (Visonic) placed above to detect entries. One of the four corners was assigned for self-stimulation (assignment balanced across rats): entries into that corner triggered laser stimulation each time the motion sensor detected movement $(25 \mathrm{~Hz} ; 15 \mathrm{~ms}$ ON $8-10 \mathrm{~mW}, 25$ ms OFF; either a 3 s pulse $(n=5)$ or a 8 s pulse $(n=4)$ for different rats). The $30 \mathrm{~min}$ session was repeated with the same corner assignment on 3 consecutive days.

In a second self-stimulation situation, Med Associates operant chambers were equipped with two empty liquid sippers on the back wall of the chamber and with grid floors wired to detect body contacts that closed a circuit with the floor. Contacts on 1 of the 2 sippers (assignment counterbalanced between rats) delivered a brief CeA laser stimulation $(25 \mathrm{~Hz}$; $15 \mathrm{~ms}$ ON, $25 \mathrm{~ms}$ OFF; either a $1 \mathrm{~s}$ train for 10 rats, similar to other optogenetic self-stimulation studies such as Witten et al., 2011) or an $8 \mathrm{~s}$ train for 4 rats (similar to duration in sucrose/salt experiments above). Contacts on the other sipper produced no consequence and served as a control measure for exploration touching or habitual spout approach. Physical contacts with the 2 empty sipper tubes were recorded over a 30 min session, and repeated for $3 \mathrm{~d}$. A separate group of rats received similar $1 \mathrm{~s}$ pulse training with the only additional feature being that contact with each sipper was accompanied by its distinctive $1 \mathrm{~s}$ sound to serve as an auditory label that contact had been achieved in case that sensory label was helpful to learn the laser versus non-laser discrimination (tone or white noise counterbalanced across spouts).

Food 'wanting': laser stimulation and general motivation to eat. We examined the effect of laser stimulation on voluntary food consumption in a $90 \mathrm{~min}$ free-intake test to determine whether the above incentiveenhancing effects of CeA stimulation on sucrose pursuit were mediated by increased appetite or general motivation to consume food rewards. Intake tests were conducted in a familiar chamber containing bedding on the floor $(38 \mathrm{~cm} \times 38 \mathrm{~cm})$ in which they had serial access to preweighed quantities of regular Purina chow pellets $(20 \mathrm{~g})$ and palatable chocolate M\&M's candies (20-25 g) while also having constant access to water. Each food intake session consisted of $30 \mathrm{~min}$ access to $20 \mathrm{~g}$ of regular chow followed by $60 \mathrm{~min}$ of access to $20-25 \mathrm{~g}$ of M\&M's candies and chow. An $8 \mathrm{~s}$ pulse of CeA laser stimulation was given once each $30 \mathrm{~s}$ period ( $25 \mathrm{~Hz} ; 15 \mathrm{~ms}$ ON, $25 \mathrm{~ms}$ OFF; $8-10 \mathrm{~mW}$; $8 \mathrm{~s}$ ON pulse duration, 22 s OFF; repeating 180 times). Intake tests were repeated on 3 consecutive days. Laser stimulation was administered only on 1 day, which occurred on either day 2 or 3 (counterbalanced across rats). Control intake was measured in the absence of any laser stimulation on the 2 remaining days (day 1 and either day 2 or 3 , averaged together to form a baseline measurement). Chow and M\&M's were reweighed at the end of the test to calculate the amount consumed.

Sucrose 'liking': laser stimulation and hedonic impact (taste reactivity). Hedonic impact was measured using a voluntary-intake taste reactivity paradigm for the taste of $45 \mathrm{mg}$ sucrose pellets (same as used for operant and Pavlovian training). Orofacial reactions elicited by sucrose pellets were video recorded using a modified voluntary ingestion model of the taste reactivity paradigm (DiFeliceantonio et al., 2012). Testing consisted 
of a single session on 2 separate days (order counterbalanced) with or without laser stimulation $(25 \mathrm{~Hz} ; 15 \mathrm{~ms}$ ON, $25 \mathrm{~ms}$ OFF). Each animal was presented with one sucrose pellet at a time ( $45 \mathrm{mg}$ ), repeated every $5 \mathrm{~s}$ for 40 times. Rats were allowed to eat voluntarily and emit postprandial hedonic reactions after each sucrose pellet. A close-up video camera beneath the transparent floor captured the image of the rat's face, mouth and paws (scoring was controlled for facial visibility). All behaviors were scored offline in slow motion (frame by frame) by an observer blinded to the conditions.

\section{Taste reactivity behaviors}

Positive hedonic 'liking' responses consisted of lateral tongue protrusions (large protrusions of the tongue extending away from the midline) and midline tongue protrusions (smaller rhythmic midline protrusions) as well as paw licking. Taste reactivity components such as tongue protrusions were scored as individual actions, whereas continuous behaviors such as paw licking were given one count for each $5 \mathrm{~s}$ bout (Berridge, 2000). Hedonic reactions were summed into affective categories to form an overall positive 'liking' score.

\section{Fos immunofluorescence and viral expression}

After training, animals were returned to the training context and given a final 30 min training session (RR6) in the presence or absence of laser stimulation. After behavioral testing, rats were deeply anesthetized with an overdose of sodium pentobarbital and perfused. Brains were then subsequently stored in $4 \%$ paraformaldehyde, cryoprotected in 30\% sucrose, and then sliced at $40 \mu \mathrm{m}$. Slices were blocked in 5\% normal donkey serum $/ 0.2 \%$ Triton-X solution for 30 min before being incubated for $24 \mathrm{~h}$ in a polyclonal goat anti-c-fos IgG primary antibody (Santa Cruz Biotechnology), followed $2 \mathrm{~d}$ later by $2 \mathrm{~h}$ in Alexa Fluor 594 donkey-antigoat IgG (Invitrogen) (Paxinos and Watson, 2007; Faure et al., 2008). Sections were mounted, air-dried, and coverslipped with ProLong Gold antifade reagent (Invitrogen). To identify fiber tip locations and assess viral spread, relevant sections were examined using a Leica microscope and results were marked on a coronal schematic in Adobe Illustrator using the rat brain atlas (Paxinos and Watson, 2007). Images were also taken using a color camera coupled to the Leica microscope at $10 \times$ and $40 \times$ magnification for Fos protein analysis. Nine images were compiled using MCID Core 7 software $(3 \times 3 ; 10 \times$ magnification $)$ into one single image centered on the fiber tip. The procedure used for measuring Fos plumes surrounding a fiberoptic site that are induced by light stimulation was modified slightly from one described previously for counting around the site of drug microinfusion (Peciña and Berridge, 2000). Immunoreactivity for Fos-like protein was visualized using a fluorescent microscopy filter with an excitation band at $515-545 \mathrm{~nm}$ for Fos-positive cells. For analysis of laser stimulation spread, Fos plume images were taken in the areas surrounding the fiber optic tip. Fos-labeled cells were individually counted within successive blocks $(50 \times 50 \mu \mathrm{m})$ along eight radial arms emanating from just under the fiber optic tip at $10 \times$ magnification. If at least two sequential blocks lacked any Fos-labeled cells, then no subsequent blocks further along the arm were counted. Zones of Fos elevation in neurons surrounding fiber optic sites (or "plumes") were assessed as described previously (Reynolds and Berridge, 2008).

\section{Statistical analysis}

Results were analyzed using repeated-measures ANOVAs to examine the response preference for either lever, followed by $t$ tests for individual comparisons. Effect sizes were calculated using Cohen's $d$. When necessary, a Wilcoxon sign-ranked test was run for nonparametric tests. For all analyses, the significance level was set at $p=0.05$, two-tailed.

\section{Results}

\section{Two-choice instrumental task}

When presented with a choice of two levers that each earned a sucrose pellet, rats trained without any amygdala laser illumination showed essentially an equal preference between the two levers and essentially selected randomly (mean 53\%: 47\% preference ratio; $\left.n=4 ; F_{(1,3)}=0.02 ; p>0.05\right)$. However, adding CeA photo-excitation associatively to the final press of one lever and to its sucrose delivery in rats with CeA channelrhodopsin (CeA ChR2 rats) caused those rats to become narrowly focused on the particular laser-associated lever by the fifth day of training, choosing and intensely pressing and nibbling on the Laser + Sucrose lever while ignoring the alternative Sucrose Alone lever that was not paired with laser $\left(t_{(1,5)}=3.01, p<0.05\right.$, Cohen's $d=$ 1.78; Fig. 2). By the eighth day, when effort levels were highest (requiring $\sim 6$ presses for each reward), CeA ChR2 rats reached over a 24:1 preference ratio to favor the laser-paired lever/reward combination over the no-laser alternative $\left(F_{(1,6)}=12.86, p<\right.$ 0.05; Fig. 2). CeA ChR2 rats also showed numerous intense consummatory actions directed specifically toward the laser-paired lever: rapidly sniffing, nibbling, and biting that particular metal object as though the laser pairing had strengthened its incentive salience properties as an attractive Pavlovian food-related cue (Video 1). Even when CeA ChR2 rats actually received their sucrose reward, they typically paused pressing and nibbling the CeA-associated lever only momentarily, just long enough to recover and consume the sucrose pellet (i.e., 1-2 s) before then immediately returning to the laser-paired lever again and resuming a frenzied bout of pressing and nibbling of the metal lever (even though no further sucrose could be earned for at least the $8 \mathrm{~s}$ timeout period while the laser was still illuminated). Therefore, CeA ChR2 rats worked 50\% harder than needed for each sucrose reward earned by the laser-paired lever (compared with 


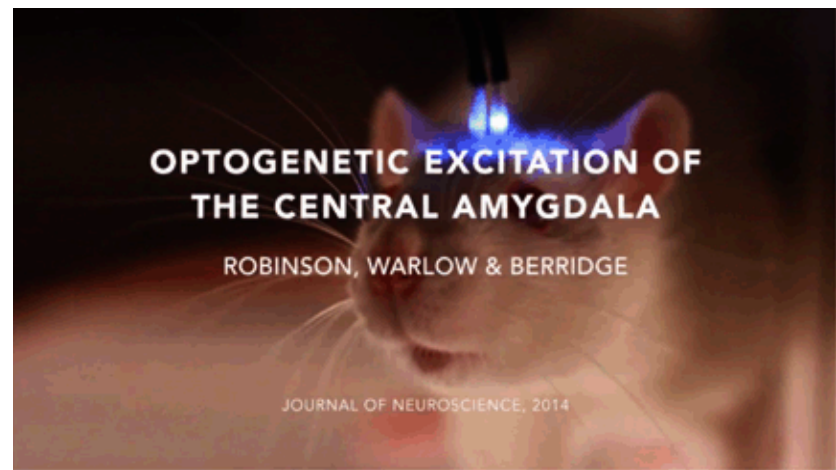

Movie 1. Example of CeA ChR2 stimulation: narrowly focused preference and amplified motivation for laser-paired sucrose reward (over equivalent sucrose reward without laser). This rat has learned to instrumentally press on two different levers, which each earned an equivalent sucrose pellet as reward. Here, CeA ChR2 laser stimulation is also paired with final press on the left lever and its sucrose reward (but not the right lever or its sucrose reward). When each lever is presented one at a time, the rat approaches, presses and nibbles both to earn rewards. Next, a simultaneous choice is offered to the rat. When both levers are presented at the same time, the rat exclusively focuses on the CeA laser-paired option (left lever). The rat returns persistently to its laser-paired lever after retrieving sucrose pellets, while ignoring the alternative right lever. This video was made after $8 \mathrm{~d}$ of discriminative training. The blue laser is visible for demonstration purposes, but equivalent $\mathrm{CeA} C \mathrm{ChR} 2$ behavioral effects have been observed when the laserheadcap connection is shielded from external view.

sucrose alone reward: $\left.F_{(1,19)}=7.34, p<0.05\right)$ and exerted nearly all of that extra effort in the few seconds while still receiving CeA illumination. In contrast, CeA ChR2 rats typically ignored the non-laser lever that earned sucrose alone, leaving it alone for up to 5-10 $\mathrm{min}$ at a time. Therefore, CeA ChR2 rats earned $>90$ pellet rewards on the CeA Laser + Sucrose lever during the 30 min session (95\% preference), compared with earning only 3 sucrose rewards (only $5 \%$ of total rewards) on the Sucrose Alone lever $\left(t_{(1,9)}=9.88, p \leq 0.001, d=4.46\right)$. The skewing of preference toward the laser-paired lever and its outcome when a simultaneous choice was given, and consequent dramatic slowing of approach to the non-laser lever, indicates that increased attraction to the Laser + Sucrose choice situation was at the expense of reduced attraction to the Sucrose Alone lever (Sucrose Alone for laser-trained vs no-laser controls: $t_{(1,16)}=3.22, p<0.01, d=$ 1.99). Further, even during the initial forced choice phase, when only one lever at a time was presented in alternating sequence, a stronger motivation to pursue the Laser + Sucrose option was evident: CeA ChR2 rats pressed 5 times faster when the Laser + Sucrose lever was available than they did when the Sucrose Alone lever was available (taking only $4 \mathrm{~s}$ to complete the required number of responses on the laser-paired lever versus averaging $21 \mathrm{~s}$ on the alternative lever; $\left.t_{(1,21)}=2.26, p<0.05, d=1.84\right)$. This difference occurred despite always initially approaching each of the two levers equally rapidly $\left(t_{(1,21)}=0.35, p>0.05\right)$ within $\sim 4$ $s$ of lever appearance in the chamber.

The CeA ChR2 laser-induced bias grew over 8 consecutive days, from an initial $62 \%$ on the first day to a $94 \%$ preference by the eighth day (Fig. 2). Preference strengthened even though the effort requirement also grew from 1 press per outcome (FR1) to a random average of 6 presses per outcome during those days (RR6; range $4-8$ presses; $F_{(7,42)}=9.86, p<0.001$ ). Preference growth probably was due primarily to time and increasing experience with Laser + Sucrose choices rather than to the additional effort requirement, because a second group $(n=4)$ of separate CeA rats kept at a lower FR1 effort demand for all $8 \mathrm{~d}$ also showed a pronounced growth in preference over days leading to at least a
5:1 preference ratio (day $\times$ laser: $F_{(7,21)}=6.03, p<0.01$; group: $\left.F_{(1,12)}=1.81, p>0.05\right)$. However on day 8 , this group's ceiling preference of $82 \%$ was still slightly lower than the $94 \%$ ceiling of the rising effort group $\left(t_{(1,12)}=2.59, p<0.05, d=1.63\right)$. Overall, all CeA ChR2 rats in both groups virtually ignored their Sucrose Alone alternative choice by the final day, suggesting an intense degree of preference and motivation focused on the laser-paired lever.

In comparison, inactive-virus control rats that similarly received CeA virus microinjections and CeA laser, but with virus that contained only the GFP gene while lacking the ChR2 gene for photoreceptors, emitted only half the number of presses on the Laser + Sucrose lever as CeA ChR2 rats $\left(F_{(1,6)}=2.63, p<0.05\right.$; Fig. 2). Inactive-virus rats showed a modest preference for their CeA laser-paired lever over their no-laser lever, suggesting either that slight reinforcing effects were exerted by light reaching the retina from external reflection or from intracranial diffusion or by other local effects of CeA illumination that did not depend on ChR2 photoreceptor expression. However, control rats with inactive-virus in CeA never pressed with the avid intensity described above for CeA ChR2 rats and instead dramatically fell below the levels of CeA ChR2 rats, especially during the last $3 \mathrm{~d}$ of testing as effort requirement rose from 4 presses on average to 6 presses on average for each reward $\left(t_{(1,34)}=2.91, p<0.01, d=\right.$ 1.04). Therefore, by the final day, inactive-virus CeA rats had only a 3:1 preference for the laser-paired lever, whereas CeA ChR2 rats had reached an $\sim 24: 1$ preference $\left(t_{(1,10)}=5.74, p<0.001, d=\right.$ 3.31).

Indicating localization of function, BLA ChR2 rats completely failed to prefer their laser-paired lever over the Sucrose Alone lever that lacked laser illumination $\left(t_{(1,7)}=0.79, p>0.05\right.$; Fig. 2$)$. If anything, the reverse: BLA ChR2 rats tended by 3:2 to prefer the non-laser lever that earned Sucrose Alone over the lever that earned Sucrose + BLA Laser. However, the BLA ChR2 preference for non-laser outcome did not reach statistical significance $\left(F_{(7,49)}=0.57, p>0.05\right)$, so future work would be required to assess whether BLA ChR2 rats express a preference or simply choose randomly. In any case, BLA ChR2 rats were far below CeA ChR2 rats in effort on the laser-paired lever, especially by the final days $\left(F_{(1,16)}=21.92, p<0.001\right)$. BLA ChR2 rats did not differ from inactive-virus CeA control rats that received illumination $\left(F_{(1,11)}=1.72, p>0.05\right)$, suggesting that both groups were similarly weak in laser effects on preference among sucrose outcomes. In contrast, CeA ChR2 rats pressed far more on the laserpaired lever by the final days than either BLA ChR2 rats $\left(t_{(1,43)}=\right.$ 5.13, $p<0.001, d=1.57)$ or CeA inactive-virus rats that also received illuminations $\left(t_{(1,34)}=2.91, p<0.01, d=1.03\right)$. That pattern indicates it was the combination of ChR2 photoreceptormediated excitation plus the CeA site for location of laser illumination that was especially crucial to produce such an intense 24:1 preference for laser-paired lever and sucrose outcome.

Finally, a narrowing in the focus of preference by CeA ChR2 laser was also evident by comparing groups' presses on the nonlaser-paired lever that earned only Sucrose Alone. By the final day, CeA ChR2 rats were actually pressing less on the non-laser lever than either CeA inactive virus control rats $\left(t_{(1,10)}=3.18\right.$, $p<0.01, d=1.83)$ or BLA ChR2 rats $\left(t_{(1,13)}=4.02, p<0.01, d=\right.$ $2.37)$ despite the generally much higher and more frenzied level of effort overall by CeA ChR2 rats. Therefore, CeA ChR2 laserpairing appeared not only to magnify but also narrowly focus all effort nearly exclusively on the sole Laser + Sucrose option at the expense of less effort being directed toward earning the alternative Sucrose Alone option. 
The failure of BLA sites to control preference or amount of lever pressing also indicates that the success of CeA sites was not due to extraneous features of the optogenetic test procedure that would have been shared by both sites (e.g., reinforcement by visible blue light diffusion from laser), but rather was specifically due to CeA optogenetic stimulation.

\section{Anatomical localization of function: CeA enhancement versus BLA failure} Histological analysis indicated that virus expression for the rats showing the incentive enhancement effects described above filled most of the medial-lateral extent of CeA (see Fig. 4). Optic fibers were placed in approximately equal proportions in medial and lateral divisions of CeA across individual rats (see Fig. 4), with the tips of fibers placed mostly in the dorsal half of $\mathrm{CeA}$ and primarily in the rostral half of the central nucleus. Observations of Fos plumes, expressed as elevations of protein in neurons surrounding an optic fiber tip after ChR2 infection, suggested that laser illumination (beginning $75 \mathrm{~min}$ before brains were extracted) activated Fos expression in surrounding neurons, primarily placed immediately below the fiber tip and extending $\sim 0.3 \mathrm{~mm}$ in radius from the fiber tip (e.g., 150\% elevation compared with $100 \%$ Fos levels observed surrounding a fiber tip in control rats that had received inactive virus but also received laser illumination; Fig. 3). The descending shape of observed Fos plumes below the tip of optic fiber in CeA rats seemed consistent with the possibility that neuronal activation might have reached into the ventral half of CeA. However, further analyses of Fos plume comparisons with other control conditions would be useful in future to confirm how deeply neuronal activation extends below a fiber tip. In any case, in CeA ChR2 rats, Fos was observed both in neurons that coexpressed virus GFP and in other neurons that were not infected by virus and which presumably were indirectly modulated across synapses via local circuit interaction. The anatomical extent of Fos spread suggested that optogenetic stimulation in CeA rats probably modulated neurons filling most of the rostral half of the CeA. Optic fiber sites were distributed in both the medial division and the lateral division of CeA and the spread of activation suggested that there also may have been some cross-division spillover; that is, a fiber in one division may have induced activation that extended also into the other division.

Given that neurons in both medial and lateral divisions of CeA were likely to be affected by many of our sites, it seems plausible to conclude that these CeA behavioral effects were primarily driven by activation of output projection neurons located in the medial division of CeA. This is because the medial division is the final common path for CeA outputs. Behavioral effects were similar across CeA rats regardless of division placement as far as could be told, and any coactivation of both lateral and medial divisions together would presumably be dominated behaviorally by the neuronal contribution originating in the medial division. In comparison, BLA sites of virus and optic fiber were located similarly dorsally, in the dorsal half of BLA, but more laterally, ventrally, and posterior to CeA sites, with BLA fiber tips clustered together in the dorsal half and caudal two-thirds of the BLA (Fig. 4). This pattern of placements suggested that BLA stimulations here may have excited neurons mostly contained in the posterior two-thirds of the BLA.

\section{CeA ChR2 laser increases breakpoint: working harder for a single outcome}

To assess independently whether CeA stimulation amplified the intensity of motivation to work on its paired lever/sucrose combination, we turned to an instrumental breakpoint or progressive ratio test of incentive motivation in which a rat faced only a single lever (Fig. 5). This test measures the breakpoint or the maximum effort price rats are willing to pay for an outcome, when the price grows progressively over a session. On one day, the available outcome was Laser + Sucrose (using the particular lever with sound label previously associated with Laser + Sucrose in the two-choice task); on another day, the outcome was Sucrose Alone without any laser (using the other lever location plus different sound previously associated with Sucrose Alone). This allowed the effect of adding CeA laser to sucrose to be assessed by comparing a rat's effort levels across the $2 \mathrm{~d}$ (order was counter- 

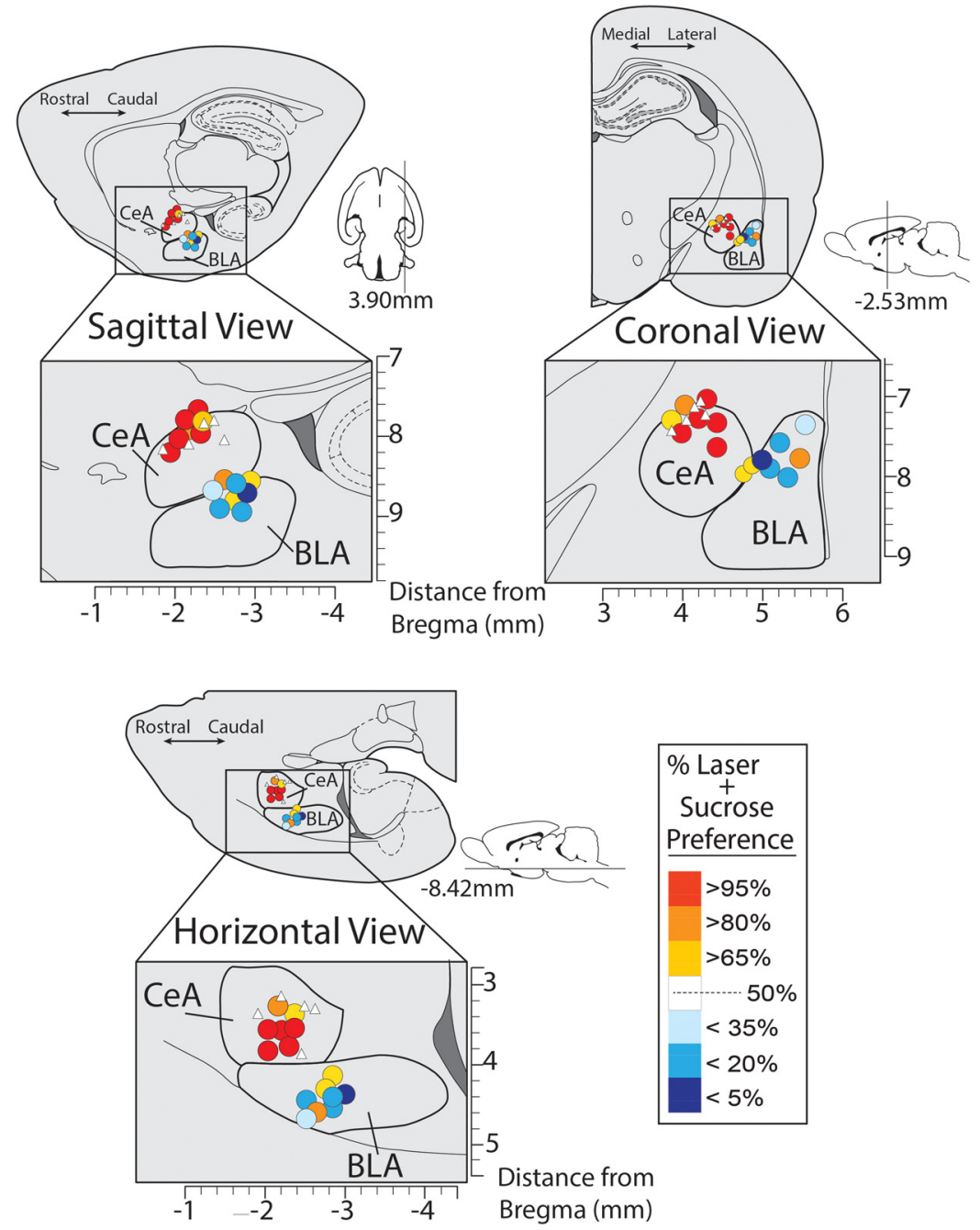

Figure 4. Localization of function maps for incentive enhancement. Maps show sites in CeA and BLA corresponding to data in Figure 2 for ChR2 enhancement of sucrose choice. Color of each symbol in map depicts the behavioral consequence of ChR2 laser stimulation at that site in the 2-sucrose choice test (\% laser preference for the Laser + Sucrose lever over the Sucrose Alone lever). Sizes of symbols from both CeA ChR2 and BLA ChR2 rats are scaled to represent the average Fos plume observations of CeA ChR2 rats who received laser, shown in Figure $3(.25 \mathrm{~mm})$. White triangles depict placements of inactive virus controls.

earned rose less than effort because the progressive ratio schedule demanded many more presses for later rewards). The CeA ChR2 laser magnification of effort actually grew in percentage terms over the 30 min session as effort demands increased, from $283 \%$ elevation in the first minutes during low initial effort (1:1 to 12:1 press: reward ratio) to $1720 \%$ elevation at the end of the session for higher effort ratios (77:1 to 200:1; interaction: $\left.F_{(1,13)}=14.75, p<0.001\right)$. Facilitation of breakpoint by CeA ChR2 laser was specific to the day it was actually illuminated: even those ChR2 rats that were tested on a first day with CeA illumination and on a subsequent day without laser illumination showed the enhancement only on the first day $\left(t_{(1,2)}=4.5 p<0.05, d=2.67\right)$ and were no longer elevated on the second day (which remained comparable to the no-laser day of other rats that were tested first in that no-laser condition; $t_{(1,5)}=$ $1.02, p>0.05)$.

In contrast, in inactive-virus control rats, CeA laser illumination failed to increase the breakpoint price and the $2 \mathrm{~d}$ did not differ in effort for sucrose $\left(t_{(1,8)}=\right.$ $0.75, p>0.05)$. Directly comparing across groups, CeA ChR2 rats pressed nearly twice as much as control CeA inactivevirus rats on their respective CeA laser day $\left(t_{(1,10)}=3.22, p<0.01, d=2.042\right)$. Therefore, CeA laser illumination made CeA ChR2 rats earn approximately twice as many sucrose pellets as control rats with $\mathrm{CeA}$ inactive-virus on the laserpaired day $\left(t_{(1,10)}=3.42, p<0.01, d=\right.$ 2.0). Finally, an additional control group of no-laser rats was tested on both days without CeA laser to assess betweensession breakpoint stability. These no-

balanced across rats). During each 30 min session, the effort requirement escalated systematically from 1 press per sucrose pellet to $>200$ presses per pellet. On the laser day, CeA laser stimulation onset began with each final instrumental press that earned a sucrose pellet and continued for $8 \mathrm{~s}$ while the sucrose was retrieved and consumed, similarly to above (Laser + Sucrose). On the non-laser day, to allow comparison with a non-laser control or baseline condition, each CeA ChR2 rat or CeA inactive-virus control rat was tested while earning sucrose pellets alone without any laser following the same progressive ratio schedule.

Breakpoint results showed that CeA ChR2 laser amplified effort to earn sucrose by $>250 \%$ compared with the same rats' performance in the baseline day that earned sucrose pellets without any laser $\left(t_{(1,12)}=3.85, p<0.01, d=2.21\right.$; Fig. $)$. CeA-ChR2 rats pressed on average up to 133 times for a single laser-paired sucrose pellet on the CeA laser day, in contrast to only 57 times for sucrose alone on the non-laser day. That is, CeA-ChR2 laser stimulation made rats press more than twice as much $\left(t_{(1,12)}=\right.$ 3.17, $p<0.01, d=1.74)$ and therefore earned $>140 \%$ more sucrose pellets than the same rats did in their Sucrose Alone session $\left(t_{(1,12)}=3.71, p<0.01, d=1.95\right.$; the number of pellets laser control rats were willing to pay on average only half (70 presses) the laser-induced CeA ChR2 breakpoint price (133 presses; $\left.t_{(1,9)}=2.87, p<0.05\right)$, which was only as much as control CeA inactive-virus rats paid on their laser day and as the CeAChR2 rats paid on their non-laser day $\left(64-76\right.$ presses; $t_{(1,6)}=$ $0.50, p>0.05)$.

\section{Reconfirmation of CeA two-choice preference}

All CeA rats were subsequently returned to the original simultaneous two-choice task in which both levers in this case easily earned the same sucrose reward (FR1) to assess the stability of their earlier CeA laser effects on choice (Laser + Sucrose vs Sucrose Alone). Illumination of the CeA laser was again paired with a final press on whichever lever it had originally been paired for each rat, and overlapped with consumption of sucrose earned on that lever ( $8 \mathrm{~s}$ duration). Under these reinstated conditions for simultaneous choice, CeA ChR2 rats still preferred their Laser + Sucrose lever/outcome option by 20:1 over the alternative of Sucrose Alone (93.9\% preference on FR1; $t_{(1,4)}=5.16, p<0.01, d=$ 11.17). This confirmed that the earlier optogenetic magnification 
of preference endured and recurred again whenever the choice was available.

\section{Making an unequal choice more equal:} salt versus sucrose

Is the power of $\mathrm{CeA} \mathrm{ChR} 2$ stimulation on preference strong enough to make a relatively unattractive outcome become as attractive as an already attractive alternative option? This question was answered by retraining CeA ChR2 rats and control CeA inactive-virus rats on a new two-choice comparison between earning either a saltadulterated pellet or a familiar sucrose pellet and assigning CeA laser pairings to the lesspreferred option. Normally, without any laser stimulation, rats preferred to earn sucrose-flavored pellets over salt-flavored pellets by a 4:1 ratio $\left(t_{(1,4)}=9.91, p<0.001\right.$; Fig. 6). We therefore designated the saltflavored pellets as the target for CeA pairing. One lever earned a 3\% salt pellet plus laser stimulation of CeA, which began with final press and continued $8 \mathrm{~s}$ while the salt pellet was consumed (Laser + Salt; 8 s illumination) similar to above. The other lever earned a sucrose pellet reward without any laser (Sucrose Alone).

Salt/sucrose results showed that associative pairing of CeA ChR2 stimulation with the earning and eating of salt-adulterated pellets magnified the incentive value and pursuit of the salt lever/outcome to make it become as attractive as sucrose by the second day, so that CeA ChR2 rats chose nearly 1:1 between the two options (53\% Sucrose Alone, $47 \%$ Laser + Salt; $t_{(1,4)}=1.37, p>0.05$; Fig. 6 , rather than $4: 1$ sucrose over salt as occurred when tested without laser. In contrast, control rats with CeA inactive-virus failed to alter preference under laser illumination and still preferred to press the Sucrose Alone lever by 5:1 over the Laser + Salt lever $\left(t_{(1,4)}=13.52, p<\right.$ $0.001, d=10.95$ ). Directly comparing the two groups, CeA laser pairing raised pressing on the salt-associated lever for salt pellets more than $8 \times$ higher in CeA Chr2 rats than in control rats with inactive-virus in $\mathrm{CeA}\left(F_{(1,8)}=10.78, p<0.05\right)$. In the CeA ChR2 rats, addition of CeA stimulation nearly doubled their number of lever presses for salt pellets compared with in the absence of CeA stimulation ( $185 \%$ elevation; $\left.F_{(1,4)}=68.93, p<0.001\right)$. Finally, when the choice was switched once more so that both levers now earned salt-adulterated pellets, but one salty outcome also earned simultaneous CeA laser stimulation (Laser + Salt), whereas the other did not (Salt Alone), preference by CeA ChR2 rats for the laser-paired salty outcome rose to become nearly 4:1 in favor of Laser + Salt over the alternative Salt Alone outcome within $3 \mathrm{~d}$ (79\% Laser + Salt; $21 \%$ Salt Alone; $t_{(1,4)}=3.5, p<0.05, d=2.59$ ), whereas no preference emerged for control CeA inactive-virus rats that chose randomly, and distributed presses equally across the two levers ( $41 \%$ Laser + Salt; $59 \%$ Salt Alone; $\left.t_{(1,4)}=2.38, p>0.05\right)$.

\section{Associative preference endures after $\mathrm{CeA}$ laser extinction}

The associative specificity described so far suggested that laser pairings made rats learn a specific preference for the designated external reward (e.g., sucrose or salt). If this preference was truly learned via associative pairings, then a CeA-induced bias should be expected to persist for some time even when the CeA laser itself was removed from the designated food out- come (i.e., imposing associative extinction conditions for laser photostimulation). To assess persistence after laser extinction, rats were returned to their originally trained choice between two sucrose options, where only one also earned CeA photostimulation (Laser + Sucrose vs Sucrose Alone) as described above, given $2 \mathrm{~d}$ retraining with additional CeA laser pairings with the rat's previously designated sucrose lever, and then put into a laser-extinction condition for the following 4 daily sessions. That is, CeA photo-excitation was removed from the previous Laser + Sucrose option, so that both levers now earned merely sucrose without laser. Results showed that CeA ChR2 rats in extinction trials continued to prefer their lever previously associated with laser by a $>20: 1$ ratio (98\% preference) over the alternative sucrose for $4 \mathrm{~d}$, even though both sucrose outcomes were now physically equal $\left(F_{(1,3)}=64.21\right.$, $p<0.01)$. They continued to press as much on their previously laser-paired lever as they had on the previous $2 \mathrm{~d}$ when CeA stimulation was still delivered $\left(F_{(3,9)}=0.76, p>0.05\right)$ without any decrease in preference or reduction of effort in extinction $\left(F_{(3,9)}=1.47, p>0.05\right)$. This persistence suggests that paired CeA stimulations had caused rats to acquire a quite robust and stable learned preference or incentive enhancement, which enduringly magnified their representation of the value of its designated lever/outcome and its pursuit.

Whether this CeA ChR2 laser-induced preference is more persistent than any other comparably strong preference induced by non-optogenetic means remains an open question. However, we surmised at least from the persistence in extinction that obtaining CeA laser stimulation itself might possibly not be the primary goal of the rats when working for an associated food reward (because laser removal produced no detectable decrement in seeking the previously designated food reward, even across 4 successive days). This possibility was tested further by investigating whether CeA ChR2 rats would work for laser stimulation in the absence of sucrose in the next experiment. 


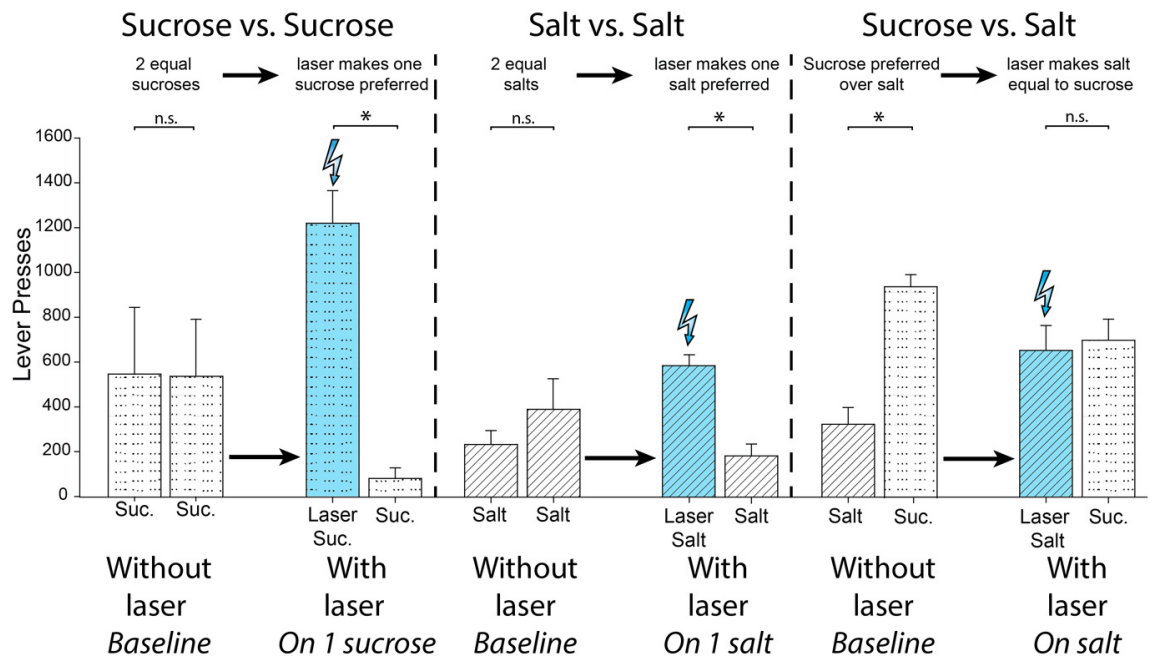

Figure 6. CeA laser enhances the relative value of its paired reward. Laser stimulation of the central amygdala transforms responding for otherwise equal rewards: sucrose versus sucrose $(n=7)$, or salt versus salt $(n=5)$ into an unequal choice. The laser-paired reward becomes strongly preferred over the reward not associated with laser stimulation. Laser stimulation of the CeA $(n=5)$ also transforms an otherwise unequal choice (sucrose vs salt) into an equal choice, making a less-preferred $3 \%$ salt adulterated pellet 'wanted' to the same degree as a sucrose pellet. Data are shown as mean \pm SEM. ${ }^{*} p<0.05$; ${ }^{* *} p<0.01$; $* * * p<0.001$.

\section{Absence of pure self-stimulation of $\mathrm{CeA}$}

To further assess whether CeA ChR2 photo-excitation itself was a goal or an independent reward or reinforcer that rats would work to gain (i.e., self-stimulate their CeA laser), we used two simple self-stimulation tasks. In these self-stimulation tasks, a rat could earn CeA photo-excitation by performing an easily acquired new action: either approaching a particular location or contacting a particular object. First, our location-approach task was similar to the original procedure used by Olds and Milner to discover the phenomenon of deep brain electrode self-stimulation reward (Olds and Milner, 1954; Milner, 1989): a rat could earn CeA ChR2 laser illumination every time it approached 1 designated corner of a 4-corner chamber. In the location-approach task, rats could obtain brief pulses of CeA laser stimulation ( $8 \mathrm{~s}$ for some rats; $3 \mathrm{~s}$ for other rats) simply by entering 1 arbitrarily designated corner of a 4-cornered square chamber in which the circular center of the chamber was occluded to encourage visiting of corners (corner assignment for CeA self-stimulation was balanced across rats, but remained consistent for each rat during testing). A pulse of CeA illumination ( 3 or $8 \mathrm{~s} ; 25 \mathrm{~Hz} ; 8-10 \mathrm{~mW}$ ) was triggered by entry into that corner, activating a positioned infrared motion detector and additional illumination pulses were earned by any further movements that activated the detector while the rat remained in that corner.

Results showed that CeA ChR2 laser stimulation failed to produce any self-stimulation or induce any preference (nor avoidance) for the paired corner on this location task $\left(F_{(3,12)}=0.53, p>0.05\right.$; Fig. $\left.7 A\right)$. No preference for the laserdelivering corner or avoidance of that corner was observed in either CeA inactive-virus rats $\left(F_{(6,24)}=0.26, p>0.05\right)$ or CeA ChR2 rats $\left(F_{(3,12)}=0.08, p>0.05\right)$ regardless of whether they earned a 3 or $8 \mathrm{~s}$ pulse of laser illumination $\left(F_{(3,7)}=0.49, p>\right.$ $0.05)$. Even CeA ChR 2 rats that had earlier robustly worked for CeA-paired sucrose at a 20:1 preference ratio failed to show any preference for their laser corner, even after 3 repeated days of testing $\left(F_{(6,24)}=0.66, p>0.05\right.$; Fig. $\left.7 A\right)$.

To confirm the lack of support for self-stimulation by CeA illumination, rats were also tested in another independent and equally simple self-administration task (designated spout-touch), which has been shown to reveal optogenetic selfstimulation of other brain systems (Kravitz et al., 2012). In this spouttouch task, rats could earn CeA laser stimulation by merely approaching and touching a particular empty metal sipper-spout inserted through a side wall of the chamber (Kravitz et al., 2012). The laser-delivering spout was always available for self-stimulation throughout the entire 30 min session and was empty though similar in appearance to water spouts in the home cage. A second empty spout was also present but earned no laser, serving as a control object for contact comparison. Each physical contact with the laserdelivering spout closed a circuit that delivered a short pulse (either 1 or $8 \mathrm{~s}$ in separate rats; $25 \mathrm{~Hz} ; 8-10 \mathrm{~mW}$ ) of laser stimulation to bilateral CeA (instrumental FR1 schedule). For some rats, each touch of the laser-designated spout was additionally tagged with a distinctive $1 \mathrm{~s}$ auditory cue to provide a further sensory label that CeA stimulation was being administered (for these rats, the other spout produced a different sound, tone, or white noise counterbalanced across rats). For rats in the no-tone condition, both spouts remained silent even when laser was delivered. All groups received 3 consecutive days of daily $30 \mathrm{~min}$ training/test sessions. Results again indicated failure to establish any self-stimulation for CeA ChR2 laser illumination (nor, conversely, any specific avoidance of CeA illumination). CeA ChR2 rats touched each spout $\sim 17$ times per session (33 times in the presence of tones), but failed to specifically touch the laser-earning spout any more or less than the alternative spout, even after three days of repeated experiences (all $F<1.38$, all $p>0.05$; Fig. $7 B$ ) regardless of whether rats were additionally informed of internal CeA ChR2 stimulation by a distinct external auditory label $\left(F_{(1,13)}=1.61, p>0.05\right)$ and whether they earned a $1 \mathrm{~s}$ pulse or an $8 \mathrm{~s}$ pulse of laser illumination $\left(F_{(1,9)}=0.32, p>0.05\right)$. Control rats with CeA inactivevirus similarly failed to show any preference or avoidance for the laser-delivering spout compared with the other spout $\left(F_{(2,26)}=\right.$ $0.001, p>0.05)$.

\section{CeA laser by itself fails to maintain Laser + Sucrose lever pressing when sucrose is removed}

However, it still remained possible that CeA stimulation might have acquired some residual independent reinforcement value or habitlike pursuit in the original simultaneous two-sucrose choice task in which one sucrose option also earned CeA laser (e.g., via association of CeA photo-excitation with its particular lever/sucrose reward; or by more extensive experience that allowed better appreciation of CeA stimulation; etc.). To answer this, we investigated whether rats which had previously learned to lever press to earn Laser + Sucrose by at least a 24:1 ratio over Sucrose Alone, would continue to work for Laser Alone on its associated lever if sucrose was removed from both levers (i.e., creating a sucrose-specific extinction condition) because they had continued when only the laser was removed. This sucrose extinction would assess whether CeA photo-excitation had any residual reward value on its own for these already-preferring rats that could at least maintain instrumental responding in an already 
well trained individual. Therefore, after establishing 2 more days of 20:1 stable responding for Laser + Sucrose versus Sucrose Alone, a sucrose extinction condition was imposed. Now, pressing on the previous Laser + Sucrose lever earned only laser alone (accompanied by its previous auditory cue), but no sucrose. Pressing on the previous Sucrose Alone lever now earned nothing (except its own previous auditory cue; auditory cues were retained as potential discriminative labels and/or conditioned reinforcers so rats would extensively sample the new outcome contingencies). To allow a comparison with more complete extinction (when nothing can be earned any longer, not even laser alone), an additional separate control group of rats $(n=4)$ were similarly trained to press and choose between Laser + Sucrose versus Sucrose Alone until they reached similar preference for Laser $+\mathrm{Su}-$ crose and then all outcomes were discontinued in complete extinction (i.e., nothing could be earned: no laser, no sucrose and no combination, although responses were still accompanied by previous auditory cues). Sucrose-extinction results showed that already-preferring rats failed to maintain lever pressing or self-stimulate at all for pure CeA photo-excitation when their associated external sucrose rewards were removed. Instead, rats reduced pressing by $2 / 3$ to a $33 \%$ level of their previous sucrose-rewarded efforts after only one session pressing for CeA stimulation alone $\left(t_{(14)}=\right.$ $3.75, p<0.01, d=1.88)$. That low effort level for CeA laser alone was equally low to the complete-extinction group's level, in which neither laser nor sucrose was earned any longer $\left(t_{(10)}=0.91, p>0.05\right)$. In other words, CeA laser-alone still appeared to be worth no more to rats than nothing at all. Both extinction groups still pressed more on their lever that had previously earned Laser + Sucrose than the alternative lever by $4: 1$ ( $t$ 's $\geq 4.67, p \leq 0.01$ ) and continued to do so over the next $7 \mathrm{~d}$ of repeated extinction $\left(F_{(1,5)}=24.29, p<0.01\right)$, indicating that their earlier-learned relative preference was highly persistent to at least a degree. However, that relative preference was not detectably influenced by whether CeA laser or nothing was actually earned during extinction and absolute effort levels were only $1 / 3$ of previous reinforced levels. Overall, these results suggest that CeA ChR2 laser by itself became essentially ineffective in further controlling behavior or choice once its associated external sucrose target was removed and that CeA photostimulation added no value of its own without an external incentive target.

\section{CeA laser stimulation fails to enhance spontaneous intake of food}

Given the apparent importance of the presence of an external sucrose reward in gating CeA amplification of incentive motivation, it seemed of interest to assess the nature of how CeA ChR2 excitation enhanced the external sucrose reward. One possibility is that CeA ChR2 stimulation might simply enhance the motivation to eat foods as unconditioned stimuli, making sucrose more attractive as an object of ingestion (i.e., 'wanting' to eat more). That is, does CeA ChR2 photo-excitation increase appetite or any direct drive to eat (whether the drive is appetitive or aversive)? A second possibility is that CeA stimulation might enhance the hedonic impact of sucrose taste. That

\section{B No self-stimulation with Sippers}

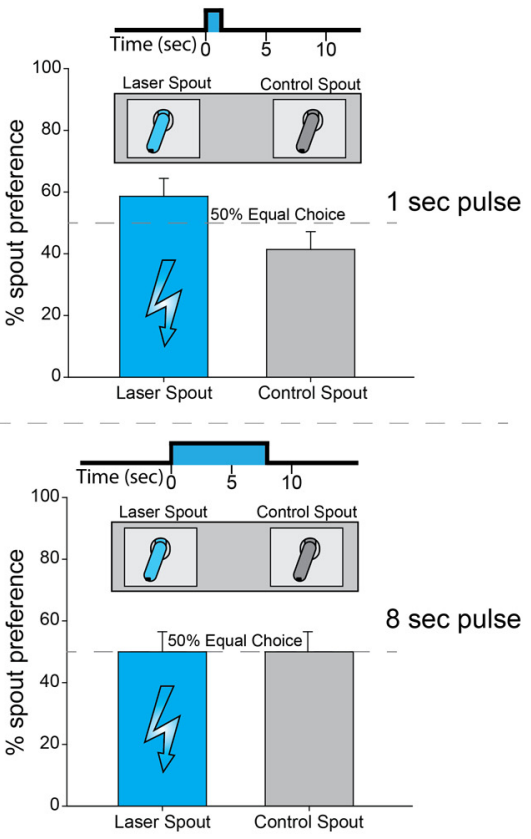

Laser Spout Control Spout

Figure 7. No self-stimulation for CeA ChR2 laser by itself. Rats would not perform easy responses to earn laser stimulation of CeA 政

is, CeA ChR2 photostimulation might make sweetness more 'liked,' which in turn might promote further 'wanting' to obtain and eat the associated reward. To assess these possibilities, first, CeA ChR2 effects on motivation to eat were assessed via a free intake test in which rats could eat as much as they wished of either of two available foods: sweet/fatty chocolate M\&Ms (each $\sim 1$ g) or ordinary Purina chow pellets during $90 \mathrm{~min}$. Each rat was tested once with CeA laser and again without laser on separate days, with order of tests balanced across rats (laser stimulation on either day 2 or 3 ). On the laser-test day, CeA laser illumination was delivered twice per min in $8 \mathrm{~s}$ bins, repeated every $\min$ for $90 \mathrm{~min}(25 \mathrm{~Hz} ; 8 \mathrm{~s} \mathrm{ON}, 22 \mathrm{~s}$ OFF per half $\mathrm{min})$. Results showed that CeA ChR2 laser stimulation failed to increase or otherwise detectably alter the intake of either chocolates or chow pellets (chow $F_{(1,4)}=0.07, p>0.05 ; \mathrm{M} \& \mathrm{M}^{\prime} \mathrm{s} F_{(1,4)}=0.31, p>$ 0.05 ; Figure $8 A$ ). Rats always ate sweetened chocolate M\&M's more than standard laboratory chow by a 9:1 ratio $\left(F_{(1,4)}=49.06, p<\right.$ $0.01)$, but CeA illumination failed to alter amounts eaten of either food. Therefore, CeA ChR2 stimulation did not simply enhance a general motivation to eat as reflected by amount consumed nor specifically to eat sweet foods.

\section{CeA ChR2 laser stimulation fails to enhance 'liking' reactions} to sucrose taste

To finally assess whether CeA influenced the hedonic impact ('liking') for sucrose taste, we measured CeA ChR2 photostimulation effects on hedonic orofacial patterns of taste reactivity elicited while eating sucrose (e.g., 'liking' reactions such as lateral tongue protrusions). We recorded orofacial reactions through a close-up video camera under the transparent floor while rats voluntarily ingested a series of $45 \mathrm{mg}$ sucrose pellets presented one at a time (40 pellets total; DiFeliceantonio et al., 2012). Each CeA ChR2 rat was tested once with CeA laser and again without laser on separate days, with order of tests balanced across rats. On the laser day, CeA optogenetic 


\section{A}

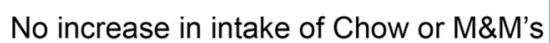

\section{B}

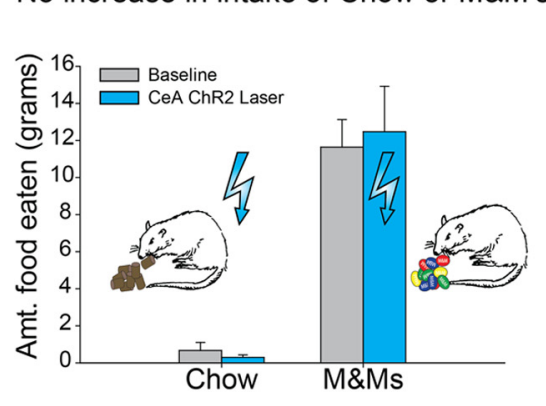

No increase in hedonic 'liking' reactions to sugar pellets

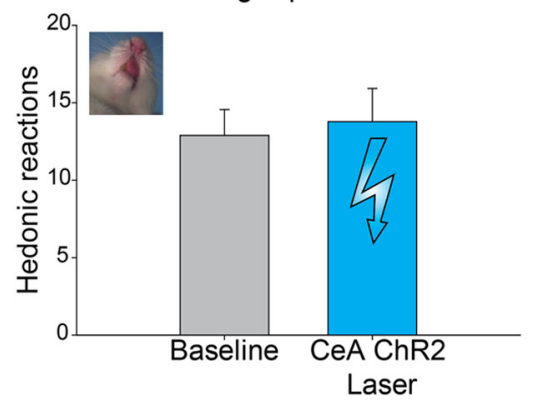

Figure 8. CeA ChR2 does not increase motivation ('wanting') to eat or the hedonic impact ('liking') of a sweet sucrose reward. $A$, CeA ChR2 laser stimulation $(n=5)$ produced no increase in the amount consumed of either chow or palatable M\&M's candies.

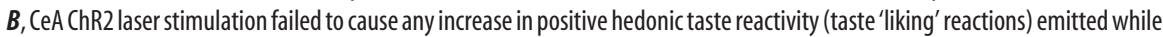
rats ate sucrose pellets $(n=9)$.

stimulation was administered throughout the session while sucrose pellets were being eaten $(25 \mathrm{~Hz} ; 15 \mathrm{~ms} \mathrm{ON}, 25 \mathrm{~ms}$ OFF), following a temporal pattern previously used for assessing increases of eating behavior induced by lateral hypothalamic electrode stimulation (Berridge and Valenstein, 1991). On the non-laser day, sucrose pellets were consumed similarly, but no CeA illumination was given. Results showed that eating of sucrose pellets was always accompanied by positive hedonic reactions (rhythmic tongue protrusions, lateral tongue protrusions or paw licking), but that CeA laser stimulation failed to enhance hedonic reactions or alter taste reactivity in any detectable way during voluntary sucrose ingestion $\left(F_{(1,8)}=0.16\right.$, $p>0.05$; Fig. $8 B$ ). CeA laser stimulation similarly failed to induce any negative aversive reactions such as gapes or headshakes, which always remained zero. Therefore, we conclude CeA ChR2 stimulation at these parameters does not strongly enhance the palatability or hedonic impact of its sweet taste, and that therefore enhanced 'liking' for sucrose reward was not the primary reason that CeA stimulation made rats 'want' to earn sucrose reward more in earlier choice and breakpoint tests of incentive motivation.

\section{Discussion}

Optogenetic (ChR2) stimulation of the CeA amplified the intensity of incentive motivation to pursue a paired external food reward. Simultaneously, CeA ChR2 stimulation narrowed the focus of that motivation to the particular act of earning sucrose that had been associatively paired with CeA photo-excitation, pulling motivation away from the alternative lever that earned comparable sucrose but lacked CeA laser. CeA ChR2 rats preferred their CeA laser-paired lever earning sucrose by 24:1 over their alternative, but otherwiseidentical Sucrose-Alone lever. When a lesser food reward (salt pellet) was paired with CeA laser to compete with a higher-value sweet reward (sucrose pellet) that lacked laser association, the laser-paired salt pellets rose to become equal in attractiveness to sucrose.

Similarly, in a separate breakpoint test of incentive motivation intensity, CeA ChR2 stimulation made rats willing to pay up to a 17-fold higher price for their sucrose reward on a progressive ratio task that required increasingly higher levels of effort as the session continued. These findings demonstrate that associative pairing of CeA photo-excitation can hijack learned choice and amplify motivation mechanisms to create a single-minded intense pursuit of the designated target.
In short, pairing with laser stimulation of the CeA seemed to have specifically amplified the motivational attractiveness or incentive value of its associatively paired reward representation (or act of earning it), raising that incentive value by at least several times. Such intense but narrow enhancement of learned motivation for a single associated target by CeA stimulation complements previous demonstrations of broader motivation effects, such as reduction of anxiety by optogenetic excitation of glutamate terminals in CeA (Tye et al., 2011) or reward-related selfstimulation and associative unblocking by optogenetic excitation of mesolimbic dopamine neurons (Kim et al., 2012; Kravitz et al., 2012; Rossi et al., 2013; Steinberg et al., 2013). Our observation of CeA ChR2 focusing of intense food-related consummatory actions, such as avid nibbling and sniffing of the associated metal lever, is also reminiscent of similar enhancements of metal Pavlovian cues into intense motivational magnets, which elicit consummatory nibbling as well as approach, by CeA opioid stimulation induced by DAMGO microinjection (Mahler and Berridge, 2009; DiFeliceantonio and Berridge, 2012). However, those pharmacological manipulations acted to intensify motivation for a previously learned reward, after learning was completed, whereas the present study used associative pairings during training to intensify learned motivation for a particular reward. Future studies will be needed to further disentangle the roles of learning versus post-learning enhancements of motivation in CeA ChR2 effects. Further studies will also be needed to identify whether it is a central representation of the paired sucrose outcome, the associated metal lever as object, or the act of earning the outcome that is the chief target for incentive enhancement by CeA ChR2 stimulation.

\section{CeA versus BLA localization of function}

Only sites within CeA for ChR2 stimulation were highly effective here in intensely enhancing incentive motivation. In contrast, ChR2 sites in BLA completely failed to have any detectable enhancement effect at all. That anatomical site difference within amygdala indicates potential localization of function to CeA for incentive motivation enhancement achieved in this way. Although BLA and CeA are serially connected and sometimes play similar roles in learned incentive motivation (Ahn and Phillips, 2003; Phillips et al., 2008; Stuber et al., 2011; Wassum et al., 2011), the two nuclei also have their own separate inputs and outputs and often play different or parallel roles (Killcross et al., 1997; Parkinson et al., 2000; Corbit and Balleine, 2005; Di Ciano and Everitt, 2005; Balleine and Killcross, 2006). Further, because sites in the medial division of $\mathrm{CeA}$, which serves as origin for CeA outputs, were as effective here as sites in the lateral CeA that projects to medial CeA, it appears plausible that activation of medial CeA output projection neurons may have been especially important in enhancing learning and incentive motivation (Ciocchi et al., 2010). This anatomical hypothesis would be valuable to test further in future studies.

Overall, our results appear consistent with suggestions that BLA and CeA can mediate qualitatively different roles in learning and motivation via parallel networks of connectivity 
(Balleine and Killcross, 2006). Similarly, macrosystem anatomical perspectives have suggested that CeA occupies a unique striatal-level within cortical-striatal-pallidal levels of forebrain organization, whereas BLA occupies a more cortical-level role (Swanson, 2005; Heimer et al., 2008). Striatal-level status may be particularly relevant to the generation of intense motivation states by the CeA site of stimulation (e.g., food-related nibbling on the laser-associated metal lever in the sucrose task; laser-specific enhancement of incentive motivation on the progressive ratio task), given that other striatal-level structures are well known to generate intense incentive motivation states (e.g., nucleus accumbens and some regions of neostriatum; Baldo and Kelley, 2007; Everitt et al., 2008; DiFeliceantonio et al., 2012; Kim et al., 2012; Peciña and Berridge, 2013; Richard et al., 2013). Other studies have similarly suggested that the CeA may contribute more than BLA to the generation of intense motivation states (Corbit and Balleine, 2005; Balleine and Killcross, 2006; Mahler and Berridge, 2009; Lee et al., 2010; DiFeliceantonio and Berridge, 2012).

Our observations of local Fos plumes surrounding the ChR2 laser sites indicated that photo-excitation in CeA succeeded in stimulating neurons located within an $\sim 0.3 \mathrm{~mm}$ radius of the optic fiber tip, perhaps especially in a ventral direction. CeA neurons within the $0.3 \mathrm{~mm}$ radius appeared to include both those directly stimulated (also expressing GFP virus, reflecting ChR2 infection) and indirectly stimulated (showing Fos but not GFP/ChR2 virus). This suggests that ChR2 directly stimulated a subset of CeA neurons that became infected by virus and that additional neurons were also recruited by laser to express Fos, such as through local synaptic circuitry interactions. Although the fiber optic tips were placed dorsally within CeA and BLA (Fig. 4), it is conceivable that the laser illumination at each tip extended more ventrally, as is consistent with our initial Fos plume analysis (Fig. 3), and is an issue that can be given future attention. The general CAG promoter used here might also have led to virus infection of other cells in CeA, including glia, so it will be of interest for future studies to more specifically examine the roles of particular CeA neuron subtypes in incentive motivation, as well as of particular anatomical point-to-point connections of CeA with other structures.

\section{CeA enhancement needs an external target (sensory reward)}

It may be an important observation that CeA ChR2 enhancement of incentive learning and motivation required the presence of an external target here (i.e., earning a sweet or salty food as sensory reward, paired with CeA stimulation). As an internal state by itself, CeA photo-excitation failed to establish any self-stimulation behavior or even to maintain the high levels of instrumental responding previously established by the combination of CeA stimulation with a designated external sucrose reward (when sucrose delivery was discontinued). Conversely, it seems noteworthy that CeA stimulation by itself was never avoided by rats in the self-stimulation test which allowed assessment of both appetitive and aversive effects (e.g., no avoidance of laser-location compared with non-laser alternatives in the place test). That suggests further that CeA stimulation here did not simply produce a strongly aversive or stressful type of internal state that motivated food seeking as an escape from distress.

Overall, our pattern of results suggests that the role of CeA photo-excitation was to selectively magnify the pursuit of the particular associated external reward. It was striking that CeA excitation produced such intense and focused enhancement of its paired sucrose or salt incentive, yet completely failed to reinforce self-stimulation to obtain the laser by itself. Although self-stimulation states can certainly be produced by optogenetic excitation of other brain structures (Rossi et al., 2013; Steinberg et al., 2014), we surmise that a function of CeA-related circuitry revealed here specifically relates to external targets (perceptual cues and associative representations of external rewards and actions). That is, CeA excitation controlled learning to 'want' particular rewards as external incentives. This does not necessarily rule out the possibility that self-stimulation someday might be produced by different CeA excitation parameters than used here, but it shows that the enhancement of food incentive value found here was not simply due to pursuit of internal CeA excitation as an independent target. Instead, our results reveal a CeA bias toward external events in the world that may be important for understanding amygdalarelated function in learning and motivation.

\section{Enhanced 'wanting', but not 'liking'}

Despite being directed at earning external rewards, such as a sucrose pellet, the CeA enhancement was limited to the incentive motivation value aspect (e.g., 'wanting' or incentive salience) of the learned reward, and did not extend to the hedonic value (e.g., 'liking' or hedonic impact) of eating actual sucrose pellets. CeA photo-excitation failed to increase the number of positive hedonic orofacial 'liking' reactions emitted as rats ate sucrose pellets. Neither did CeA stimulation simply seem to enhance food as a target of unconditioned eating, in the sense of broadly increasing appetite or the motivation to ingest. CeA ChR2 excitation failed to make rats eat any more of either a sweet food (chocolates) or regular food (chow pellets). Lack of eating facilitation contrasts with the enhancements of incentive salience previously reported for pharmacological opioid stimulation of the CeA by microinjections of a mu opioid agonist, which not only increase the incentive salience of a Pavlovian CS + for food reward, but actually increase intake of the unconditioned food too (Gosnell, 1988; Will et al., 2004; Mahler and Berridge, 2009; DiFeliceantonio and Berridge, 2012). This CeA intake difference remains to be fully explained, but might be related to the recent identification of a subpopulation of CeA GABA-ergic neurons expressing protein kinase $\mathrm{C}$ that exert hypophagic effects when activated, which may have been stimulated by CeA ChR2 photo-excitation here (Cai et al., 2014). Finally, our failure to find increases in hedonic impact or food intake does not necessarily rule out the possibility that future optogenetic studies might find enhancement of 'liking' or 'wanting' to eat by different CeA optogenetic parameters, but does indicate again that neither of those functions explains the dramatic elevation of learned external incentive seeking and hijacking of choice demonstrated here (and we note that lack of hedonic enhancement is also consistent with previous reports that CeA pharmacological stimulations that enhance 'wanting' do so without necessarily altering 'liking,' even for mu opioid agonist microinjections, and conversely that CeA lesions that disrupt 'wanting' need not impair 'liking' for the same ingestive reward (Seeley et al., 1993; Mahler and Berridge, 2009; Riley and King, 2013).

\section{Conclusion}

CeA-induced amplification and focusing of intense incentive motivation in a narrow concentration upon one particular 
reward made the associated external target become avidly desired in a winner-take-all manner. Exclusivity of intense learned 'wanting' can be adaptive when appropriately regulated (e.g., by hunger versus thirst), but also is a signature feature of pathological drug addiction, binge eating, gambling, and related disorders in which similarly intense motivation to pursue one individualized reward (e.g., a drug of abuse) occurs at the expense of pursuing alternative life goals (e.g., family, health, or career). Our findings suggest that activating CeA-related circuitry in paired association with a particular reward may be a potential mechanism that boosts the intensity and narrowness of a learned preference into the pathological range, potentially relevant to addictions (Lu et al., 2005; Wrase et al., 2008; Lesscher and Vanderschuren, 2012).

\section{References}

Ahn S, Phillips AG (2003) Independent modulation of basal and feedingevoked dopamine efflux in the nucleus accumbens and medial prefrontal cortex by the central and basolateral amygdalar nuclei in the rat. Neuroscience 116:295-305. CrossRef Medline

Baldo BA, Kelley AE (2007) Discrete neurochemical coding of distinguishable motivational processes: insights from nucleus accumbens control of feeding. Psychopharmacology (Berl) 191:439-459. CrossRef Medline

Balleine BW, Killcross S (2006) Parallel incentive processing: an integrated view of amygdala function. Trends Neurosci 29:272-279. CrossRef Medline

Baxter MG, Murray EA (2002) The amygdala and reward. Nat Rev Neurosci 3:563-573. CrossRef Medline

Berridge KC (2000) Measuring hedonic impact in animals and infants: microstructure of affective taste reactivity patterns. Neurosci Biobehav Rev 24:173-198. CrossRef Medline

Berridge KC, Valenstein ES (1991) What psychological process mediates feeding evoked by electrical stimulation of the lateral hypothalamus? Behav Neurosci 105:3-14. CrossRef Medline

Cai H, Haubensak W, Anthony TE, Anderson DJ (2014) Central amygdala PKC- $\delta+$ neurons mediate the influence of multiple anorexigenic signals. Nat Neurosci 17:1240-1248. CrossRef Medline

Ciocchi S, Herry C, Grenier F, Wolff SB, Letzkus JJ, Vlachos I, Ehrlich I, Sprengel R, Deisseroth K, Stadler MB, Müller C, Lüthi A (2010) Encoding of conditioned fear in central amygdala inhibitory circuits. Nature 468:277-282. CrossRef Medline

Corbit LH, Balleine BW (2005) Double dissociation of basolateral and central amygdala lesions on the general and outcome-specific forms of Pavlovian-instrumental transfer. J Neurosci 25:962-970. CrossRef Medline

Di Ciano P, Everitt BJ (2005) Neuropsychopharmacology of drug seeking: Insights from studies with second-order schedules of drug reinforcement. Eur J Pharmacol 526:186-198. CrossRef Medline

DiFeliceantonio AG, Berridge KC (2012) Which cue to "want?" Opioid stimulation of central amygdala makes goal-trackers show stronger goaltracking, just as sign-trackers show stronger sign-tracking. Behav Brain Res 230:399-408. CrossRef Medline

DiFeliceantonio AG, Mabrouk OS, Kennedy RT, Berridge KC (2012) Enkephalin surges in dorsal neostriatum as a signal to eat. Curr Biol 22: 1918-1924. CrossRef Medline

Everitt BJ, Belin D, Economidou D, Pelloux Y, Dalley JW, Robbins TW (2008) Neural mechanisms underlying the vulnerability to develop compulsive drug-seeking habits and addiction. Philos Trans R Soc Lond B Biol Sci 363:3125-3135. CrossRef Medline

Faure A, Reynolds SM, Richard JM, Berridge KC (2008) Mesolimbic dopamine in desire and dread: enabling motivation to be generated by localized glutamate disruptions in nucleus accumbens. J Neurosci 28:7184-7192. CrossRef Medline

Gosnell BA (1988) Involvement of mu opioid receptors in the amygdala in the control of feeding. Neuropharmacology 27:319-326. CrossRef Medline

Heimer L, Van Hoesen GW, Trimble M, Zahm DS (2008) Anatomy of neuropsychiatry: the new anatomy of the basal forebrain and its implications for neuropsychiatric illness. New York: Elsevier.

Killcross S, Robbins TW, Everitt BJ (1997) Different types of fear- conditioned behaviour mediated by separate nuclei within amygdala. $\mathrm{Na}-$ ture 388:377-380. CrossRef Medline

Kim KM, Baratta MV, Yang A, Lee D, Boyden ES, Fiorillo CD (2012) Optogenetic mimicry of the transient activation of dopamine neurons by natural reward is sufficient for operant reinforcement. PLoS One 7:e33612. CrossRef Medline

Kravitz AV, Tye LD, Kreitzer AC (2012) Distinct roles for direct and indirect pathway striatal neurons in reinforcement. Nat Neurosci 15:816-818. CrossRef Medline

LeDoux J (2007) The amygdala. Curr Biol 17:R868-R874. CrossRef Medline

Lee HJ, Gallagher M, Holland PC (2010) The central amygdala projection to the substantia nigra reflects prediction error information in appetitive conditioning. Learn Mem 17:531-538. CrossRef Medline

Lesscher HM, Vanderschuren LJ (2012) Compulsive drug use and its neural substrates. Rev Neurosci 23:731-745. CrossRef Medline

Lingawi NW, Balleine BW (2012) Amygdala central nucleus interacts with dorsolateral striatum to regulate the acquisition of habits. J Neurosci 32:1073-1081. CrossRef Medline

Lu L, Dempsey J, Shaham Y, Hope BT (2005) Differential long-term neuroadaptations of glutamate receptors in the basolateral and central amygdala after withdrawal from cocaine self-administration in rats. J Neurochem 94:161-168. CrossRef Medline

Mahler SV, Berridge KC (2009) Which cue to "want?" Central amygdala opioid activation enhances and focuses incentive salience on a prepotent reward cue. J Neurosci 29:6500-6513. CrossRef Medline

Makris N, Gasic GP, Seidman LJ, Goldstein JM, Gastfriend DR, Elman I, Albaugh MD, Hodge SM, Ziegler DA, Sheahan FS, Caviness VS Jr, Tsuang MT, Kennedy DN, Hyman SE, Rosen BR, Breiter HC (2004) Decreased absolute amygdala volume in cocaine addicts. Neuron 44:729-740. CrossRef Medline

Milner PM (1989) The discovery of self-stimulation and other stories. Neurosci Biobehav Rev 13:61-67. CrossRef Medline

Olds J, Milner PM (1954) Positive reinforcement produced by electrical stimulation of septal area and other regions of rat brain. J Comp Physiol Psychol 47:419-427. CrossRef Medline

Parkinson JA, Robbins TW, Everitt BJ (2000) Dissociable roles of the central and basolateral amygdala in appetitive emotional learning. Eur J Neurosci 12:405-413. CrossRef Medline

Paxinos G, Watson C (2007) The rat brain in stereotaxic coordinates, Ed 6 . New York: Elsevier.

Peciña S, Berridge KC (2000) Opioid site in nucleus accumbens shell mediates eating and hedonic "liking" for food: map based on microinjection Fos plumes. Brain Res 863:71-86. CrossRef Medline

Peciña S, Berridge KC (2013) Dopamine or opioid stimulation of nucleus accumbens similarly amplify cue-triggered "wanting" for reward: entire core and medial shell mapped as substrates for PIT enhancement. Eur J Neurosci 37:1529-1540. CrossRef Medline

Pelloux Y, Murray JE, Everitt BJ (2013) Differential roles of the prefrontal cortical subregions and basolateral amygdala in compulsive cocaine seeking and relapse after voluntary abstinence in rats. Eur J Neurosci. In press.

Phillips AG, Vacca G, Ahn S (2008) A top-down perspective on dopamine, motivation and memory. Pharmacol Biochem Behav 90:236-249. CrossRef Medline

Reynolds SM, Berridge KC (2008) Emotional environments retune the valence of appetitive versus fearful functions in nucleus accumbens. Nat Neurosci 11:423-425. CrossRef Medline

Richard JM, Castro DC, Difeliceantonio AG, Robinson MJ, Berridge KC (2013) Mapping brain circuits of reward and motivation: in the footsteps of Ann Kelley. Neurosci Biobehav Rev 37:1919-1931. CrossRef Medline

Richardson NR, Roberts DC (1996) Progressive ratio schedules in drug selfadministration studies in rats: a method to evaluate reinforcing efficacy. J Neurosci Methods 66:1-11. CrossRef Medline

Riley CA, King MS (2013) Differential effects of electrical stimulation of the central amygdala and lateral hypothalamus on Fos-immunoreactive neurons in the gustatory brainstem and taste reactivity behaviors in conscious rats. Chem Senses 38:705-717. CrossRef Medline

Roesch MR, Esber GR, Li J, Daw ND, Schoenbaum G (2012) Surprise! Neural correlates of Pearce-Hall and Rescorla-Wagner coexist within the brain. Eur J Neurosci 35:1190-1200. CrossRef Medline

Rossi MA, Sukharnikova T, Hayrapetyan VY, Yang L, Yin HH (2013) Op- 
erant self-stimulation of dopamine neurons in the substantia nigra. PLoS One 8:e65799. CrossRef Medline

Saunders BT, Robinson TE (2011) Individual variation in the motivational properties of cocaine. Neuropsychopharmacology 36:1668-1676. CrossRef Medline

Schneider F, Habel U, Wagner M, Franke P, Salloum JB, Shah NJ, Toni I, Sulzbach C, Hönig K, Maier W, Gaebel W, Zilles K (2001) Subcortical correlates of craving in recently abstinent alcoholic patients. Am J Psychiatry 158:1075-1083. CrossRef Medline

Seeley RJ, Galaverna O, Schulkin J, Epstein AN, Grill HJ (1993) Lesions of the central nucleus of the amygdala II: Effects on intraoral $\mathrm{NaCl}$ intake. Behav Brain Res 59:19-25. CrossRef Medline

Steinberg EE, Keiflin R, Boivin JR, Witten IB, Deisseroth K, Janak PH (2013) A causal link between prediction errors, dopamine neurons and learning. Nat Neurosci 16:966-973. CrossRef Medline

Steinberg EE, Boivin JR, Saunders BT, Witten IB, Deisseroth K, Janak PH (2014) Positive reinforcement mediated by midbrain dopamine neurons requires $\mathrm{d} 1$ and $\mathrm{d} 2$ receptor activation in the nucleus accumbens. PLoS One 9:e94771. CrossRef Medline

Stuber GD, Sparta DR, Stamatakis AM, van Leeuwen WA, Hardjoprajitno JE, Cho S, Tye KM, Kempadoo KA, Zhang F, Deisseroth K, Bonci A (2011) Excitatory transmission from the amygdala to nucleus accumbens facilitates reward seeking. Nature 475:377-380. CrossRef Medline

Swanson LW (2005) Anatomy of the soul as reflected in the cerebral hemi- spheres: neural circuits underlying voluntary control of basic motivated behaviors. J Comp Neurol 493:122-131. CrossRef Medline

Tye KM, Prakash R, Kim SY, Fenno LE, Grosenick L, Zarabi H, Thompson KR, Gradinaru V, Ramakrishnan C, Deisseroth K (2011) Amygdala circuitry mediating reversible and bidirectional control of anxiety. Nature 471:358-362. CrossRef Medline

Wassum KM, Cely IC, Balleine BW, Maidment NT (2011) Micro-opioid receptor activation in the basolateral amygdala mediates the learning of increases but not decreases in the incentive value of a food reward. J Neurosci 31:1591-1599. CrossRef Medline

Will MJ, Franzblau EB, Kelley AE (2004) The amygdala is critical for opioidmediated binge eating of fat. Neuroreport 15:1857-1860. CrossRef Medline

Witten IB, Steinberg EE, Lee SY, Davidson TJ, Zalocusky KA, Brodsky M, Yizhar O, Cho SL, Gong S, Ramakrishnan C, Stuber GD, Tye KM, Janak PH, Deisseroth K (2011) Recombinase-driver rat lines: tools, techniques, and optogenetic application to dopamine-mediated reinforcement. Neuron 72:721-733. CrossRef Medline

Wrase J, Makris N, Braus DF, Mann K, Smolka MN, Kennedy DN, Caviness VS, Hodge SM, Tang L, Albaugh M, Ziegler DA, Davis OC, Kissling C, Schumann G, Breiter HC, Heinz A (2008) Amygdala volume associated with alcohol abuse relapse and craving. Am J Psychiatry 165:1179-1184. CrossRef Medline 